Canad. Math. Bull. Vol. 21 (3), 1978

\title{
THE CHINESE REMAINDER THEOREM AND THE INVARIANT BASIS PROPERTY
}

\author{
DAVID F. ANDERSON
}

\begin{abstract}
The Chinese Remainder Theorem states that if $I$ and $J$ are comaximal ideals of a ring $R$, then $A /(I \cap J) A$ is isomorphic to $A / I A \times A / J A$ for any left $R$-module $A$. In this paper we study the converse; when does $A /(I \cap J) A$ and $A / I A \times A / J A$ isomorphic imply that $I$ and $J$ are comaximal?
\end{abstract}

One of the most useful tools in ring theory is the Chinese Remainder Theorem (CRT): if $I$ and $J$ are ideals of a ring $R$ (with 1 ) which are comaximal $(I+J=R)$, then the natural homomorphism $R \rightarrow R / I \times R / J$ induces an isomorphism $f: R /(I \cap J) \rightarrow R / I \times R / J$. $f$ is both a ring and $R$-module isomorphism. More generally, if $A$ is any left $R$-module, the natural homomorphism $A /(I \cap J) A \rightarrow A / I A \times A / J A$ is an isomorphism. We remark that CRT fails if $I$ and $J$ are only assumed to be comaximal left ideals.

A natural question arises: if $A /(I \cap J) A$ and $A / I A \times A / J A$ are isomorphic (not necessarily by the natural homomorphism), does $I+J=R$ ? We say that a $R$-module $A$ satisfies CC1 if whenever $A /(I \cap J) A$ and $A / I A \times A / J A$ are isomorphic, then $I+J=R$. A module need not satisfy $\mathrm{CC} 1$; for example, if $F$ is a free $R$-module of infinite rank, then $F \approx F \times F$, so $\mathrm{CC} 1$ fails for $F$ with $I=J=0$. Also, the $\mathbb{Z}$-module $\mathbb{Z} / 2 \mathbb{Z}$ does not satisfy CC1 with $I=J=3 \mathbb{Z}$.

We first consider the case when $R$ is commutative. Recall that a $R$-module $A$ is locally finitely generated if $A_{M}$ is a finitely generated $R_{M}$-module for all maximal ideals $M$ of $R$. $J(R)$ will denote the Jacobson radical of $R$.

Proposition 1. Let $R$ be a commutative ring and $A$ a $R$-module.

(1) If $A$ satisfies $\mathrm{CC} 1$, then $A / M A$ is a finitely generated $R$-module for all maximal ideals $M$.

(2) Assume that $A$ is locally finitely generated, then $A$ satisfies CC1 iff $A_{M} \neq 0$ for all maximal ideals $M$.

(3) If $A$ is locally finitely generated, then $A$ satisfies CC1 implies ann $(A) \subset$ $J(R)$. If $A$ is finitely generated, then $A$ satisfies $\mathrm{CC1}$ iff $\operatorname{ann}(A) \subset J(R)$.

Proof. (1) If some $V=A / M A$ is not finitely generated, then $V$ is an infinite dimensional vector space over $k=R / M$. Thus $V \approx V \times V$ as $k$-modules, and hence as $R$-modules. Thus CC1 fails for $A$ with $I=J=M$.

Received by the editors August 3, 1977.

AMS(MOS) subject classification (1970). Primary 13A99, 16A48. 
(2) Suppose there is an isomorphism $f: A /(I \cap J) A \rightarrow A / I A \times A / J A$ with $I+J \neq R$; then $I+J$ is contained in some maximal ideal $M$. Let $N=$ $A_{M} / M_{M} A_{M}$, then $f$ induces an isomorphism $\bar{f}: N \rightarrow N \times N$. Since $N$ is a finitely generated $R_{M} / M_{M}$ vector space, necessarily $N=0$. But thus $A_{M}=0$ by Nakayama's Lemma. Conversely, if some $A_{M}=0$, then $A / M A=0$, so $A / M A \approx A / M A \times A / M A$. Thus CC1 fails for $A$ with $I=J=M$.

(3) This follows from (2) because $A_{M} \neq 0$ implies ann(A) $\subset M$. If $A$ is finitely generated then $A_{M} \neq 0$ iff $\operatorname{ann}(A) \subset M$.

(3) shows that the converse of (1) need not hold. Let $P$ be the set of prime numbers, then $A=\mathbb{Q} \oplus \sum_{p \in p} \mathbb{Z} / p \mathbb{Z}$ is not locally finitely generated, but $A$ satisfies CC1. Over a local ring any finitely generated module satisfies CC1. Any free $R$-module of finite rank satisfies $C C 1$. Let $Q$ be the set of odd prime numbers, then $A=\sum_{q \in Q} \mathbb{Z} / q \mathbb{Z}$ is locally finitely generated, has $\operatorname{ann}(A)=0$, but does not satisfy CC1. Hence the converse of the first part of (3) does not hold.

A related question is: which rings $R$ satisfy $\mathrm{CC} 1$ for all finitely generated free $R$-modules? Thus we say that a ring $R$ satisfies $\mathrm{CC} 2$ if all finitely generated free left $R$-modules satisfy $\mathrm{CC} 1$. Proposition 1 shows that any commutative ring satisfies $\mathrm{CC} 2$.

We recall that a ring $R$ satisfies the invariant basis property or invariant basis number (IBN) if $R^{m} \approx R^{n}$ implies $m=n$. Rings which satisfy IBN include commutative rings, division rings, and (left) noetherian rings. Let $k$ be a field and $V$ an infinite dimensional $k$ vector space, then $R=\operatorname{Hom}_{k}(V, V)$ does not satisfy IBN. An excellent reference on the invariant basis property is [1].

Proposition 2. A ring $R$ satisfies CC2 iff every homomorphic image of $\boldsymbol{R}$ satisfies IBN.

Proof. Suppose that some $\bar{R}=R / L$ does not satisfy IBN; then $\bar{R}^{m} \approx \bar{R}^{n}$ for some $m<n$. Choose $i, j \geq 0$ so that $i(n-m)=m+j$, then $\bar{R}^{m+i} \approx \bar{R}^{m+i(n-m)+j}=$ $\bar{R}^{2(m+j)}$. Let $l=m+j$, then $\bar{R}^{l} \approx \bar{R}^{l} \times \bar{R}^{l}$; so $\mathrm{CCl}$ fails for $R^{l}$ with $I=J=L$.

Conversely, suppose CC2 fails. Then there are ideals $I$ and $J$ with $I+J \neq R$ and a finitely generated free $R$-module $F$ such that $F /(I \cap J) F$ and $F / I F \times F / J F$ are isomorphic, by say $f$. Let $L=I+J$, then $f$ induces an isomorphism $\bar{f}$ : $F / L F \rightarrow F / L F \times F / L F$. Thus $\bar{R}=R / L$ does not satisfy IBN.

Thus any ring which satisfies CC2 also satisfies IBN. However, the converse is not true. For there exists a ring $R$ which satisfies IBN, but not all of its homomorphic images satisfy IBN [1, p. 221]. Thus the class of rings which satisfy CC2 lies strictly between the class of commutative rings and the class of rings which satisfy IBN.

\section{REFERENCES}

1. P. M. Cohn, Some remarks on the invariant basis property, Topology, 5 (1966), 215-228.

DepartMent of MATHEMATICS

University of TenNessee, KNoXville, Tennessee 37916 